\title{
Kinematic and diabatic vertical velocity climatologies from a chemistry climate model
}

\author{
Charlotte Marinke Hoppe ${ }^{1, a, b}$, Felix Ploeger ${ }^{1}$, Paul Konopka ${ }^{1}$, and Rolf Müller ${ }^{1}$ \\ ${ }^{1}$ Institute of Energy and Climate Research (IEK-7), Forschungszentrum Jülich GmbH, Jülich, Germany \\ ${ }^{a}$ now at: Institute of Energy and Climate Research (IEK-8), Forschungszentrum Jülich GmbH, Jülich, Germany \\ ${ }^{b}$ now at: Rhenish Institute for Environmental Research, University of Cologne, Cologne, Germany \\ Correspondence to: Charlotte Marinke Hoppe (c.hoppe@fz-juelich.de)
}

Received: 28 September 2015 - Published in Atmos. Chem. Phys. Discuss.: 2 November 2015

Revised: 2 May 2016 - Accepted: 6 May 2016 - Published: 23 May 2016

\begin{abstract}
The representation of vertical velocity in chemistry climate models is a key element for the representation of the large-scale Brewer-Dobson circulation in the stratosphere. Here, we diagnose and compare the kinematic and diabatic vertical velocities in the ECHAM/Modular Earth Submodel System (MESSy) Atmospheric Chemistry (EMAC) model. The calculation of kinematic vertical velocity is based on the continuity equation, whereas diabatic vertical velocity is computed using diabatic heating rates. Annual and monthly zonal mean climatologies of vertical velocity from a 10-year simulation are provided for both kinematic and diabatic vertical velocity representations. In general, both vertical velocity patterns show the main features of the stratospheric circulation, namely, upwelling at low latitudes and downwelling at high latitudes. The main difference in the vertical velocity pattern is a more uniform structure for diabatic and a noisier structure for kinematic vertical velocity. Diabatic vertical velocities show higher absolute values both in the upwelling branch in the inner tropics and in the downwelling regions in the polar vortices. Further, there is a latitudinal shift of the tropical upwelling branch in boreal summer between the two vertical velocity representations with the tropical upwelling region in the diabatic representation shifted southward compared to the kinematic case. Furthermore, we present mean age of air climatologies from two transport schemes in EMAC using these different vertical velocities and analyze the impact of residual circulation and mixing processes on the age of air. The age of air distributions show a hemispheric difference pattern in the stratosphere with younger air in the Southern Hemisphere and older air in the Northern Hemisphere using the trans-
\end{abstract}

port scheme with diabatic vertical velocities. Further, the age of air climatology from the transport scheme using diabatic vertical velocities shows a younger mean age of air in the inner tropical upwelling branch and an older mean age in the extratropical tropopause region.

\section{Introduction}

The numerical representation of vertical velocity in meteorological models can be established in various ways. The implemented vertical velocity representation depends on the vertical grid structure of the model. Various coordinate systems can be used to define vertical model layers, such as pressure $p$ or potential temperature $\theta$, with respective vertical velocities $\omega=\frac{\mathrm{D} p}{\mathrm{D} t}$ and $\dot{\theta}=\frac{\mathrm{D} \theta}{\mathrm{D} t}$ (e.g., Kasahara, 1974). Hence, in chemistry climate models (CCMs), different vertical velocity representations may be used for the advection of chemical trace gases, a fact which needs to be considered when comparing modeled trace gas distributions.

If a pressure-based vertical coordinate system is implemented, the associated vertical velocity $\omega$ is calculated as a residual from the horizontal flux divergence using the continuity equation. This method is denoted kinematic vertical velocity representation and most commonly used in CCMs.

The potential temperature $\theta$ can also be used as the vertical coordinate in a model, forming isentropic vertical model layers. Usage of $\theta$ is especially suitable in the stratosphere, where the flow mainly propagates along isentropic surfaces (e.g., Danielsen, 1961; McKenna et al., 2002b; Mahowald et al., 2002). In this configuration, vertical velocities are de- 
rived from diabatic heating rates. The corresponding vertical velocity $\dot{\theta}$ is referred to as diabatic vertical velocity.

In a perfect model, all vertical velocity representations would deliver the same result. However, inaccuracies are always present in numerical models. They occur due to numerical discretization of the underlying equations, limited accuracy of representation of numbers in computers, and parametrizations of sub-grid scale processes. These inaccuracies lead to differences in vertical velocity fields when using different vertical velocity representations. There are typical patterns that occur in the vertical velocity distributions of the aforementioned numerical representations. One example is noisy small-scale structures in the kinematic vertical velocity field, as reported by Schoeberl et al. (2003) and Ploeger et al. (2011), although their results also contain some effects from the data assimilation scheme.

The horizontal discretization also has an impact on the simulated vertical velocity field. In this study we consider the chemistry climate model ECHAM/Modular Earth Submodel System (MESSy) Atmospheric Chemistry (EMAC; Röckner et al., 2006; Jöckel et al., 2010) and find that the vertical velocity may even differ between the dynamics and the transport scheme in the same CCM. In the EMAC model, the tracer transport is calculated on a regular grid structure, while the model dynamics is calculated in spectral representation. Consequently, the vertical velocity used for tracer transport differs from the vertical velocity in the dynamical core.

It is difficult to validate model results for large-scale, stratospheric vertical velocity, as this quantity cannot be measured directly. In the atmosphere, vertical velocities are much smaller than horizontal velocities, except for fast convection events. Thus, modeled vertical velocity can only be compared to the vertical velocity from reanalyses, like ERA-Interim (Dee et al., 2011) from the European Centre for Medium-Range Weather Forecasts (ECMWF). However, vertical velocities in reanalysis themselves suffer from large inaccuracies (e.g., Abalos et al., 2015).

To overcome the problem of observability of vertical velocity, trace gas observations from satellite remote sensing instruments are compared to modeled trace gas distributions. However, the interpretation of the differences of the distributions should be handled with care, since those tracer distributions result from several different processes in the atmosphere, namely, advective transport, mixing, and chemical reactions. In particular, for mean age of air (the average transit time of an air parcel through the stratosphere) both advective transport and mixing are involved (Garny et al., 2014; Ploeger et al., 2015). Thus, precise knowledge of the vertical velocity is crucial for the analysis of stratospheric trace gas and age of air distributions to distinguish between advection and mixing effects. Considering only trace gas distributions does not allow for residual transport and mixing to be differentiated.

This work presents diagnostics to obtain the vertical velocity of the tracer transport scheme in the CCM EMAC (Röck- ner et al., 2006; Jöckel et al., 2010), and in the coupled model system EMAC-CLaMS (Chemical Lagrangian Model of the Stratosphere) (Hoppe et al., 2014) in Sect. 2. Monthly and annual zonal mean climatologies of kinematic and diabatic vertical velocities in EMAC are shown and the characteristics of each vertical velocity representation are discussed in Sect. 3. The influences of the vertical velocity on age of air distributions are investigated and the possibilities and limitations of the mean age of air diagnostic are discussed in Sect. 4. Conclusions are given in Sect. 5.

\section{Theory: vertical velocity representations}

This section describes the calculation of the kinematic and diabatic vertical velocity in the framework of the coupled model system EMAC-CLaMS (Hoppe et al., 2014). This model system consists of the EMAC (Röckner et al., 2006; Jöckel et al., 2010) and the CLaMS (McKenna et al., 2002a, b; Konopka et al., 2004; Grooß et al., 2005; Pommrich et al., 2014). EMAC-CLaMS contains diagnostics for kinematic and diabatic vertical velocities to serve as input to the tracer transport scheme. The two vertical velocity diagnostics are calculated simultaneously in grid-point space during the same model run; thus, the model setup such as radiation, trace gases for radiation input, and resolution of the model grid, are identical.

\subsection{Kinematic vertical velocity}

The standard vertical velocity in EMAC is derived from the spectral advection scheme in ECHAM5. The vertical wind $\dot{\eta}=\frac{\mathrm{D} \eta}{\mathrm{D} t}$ in ECHAM5 is calculated from the zonal and meridional horizontal winds using the continuity equation:

$$
\frac{\partial}{\partial \eta}\left(\frac{\partial p}{\partial t}\right)+\nabla \cdot\left(v_{\mathrm{h}} \frac{\partial p}{\partial \eta}\right)+\frac{\partial}{\partial \eta}\left(\dot{\eta} \frac{\partial p}{\partial \eta}\right)=0 .
$$

Here, $\eta$ denotes the terrain following hybrid pressurebased vertical coordinate in ECHAM5 (see Roeckner et al., 2003). $v_{\mathrm{h}}$ is the horizontal wind vector on an ECHAM5 model layer and $\nabla$ the horizontal gradient operator. After the advection time step, the new surface pressure is calculated for each grid box, which determines the pressure levels of the hybrid model grid for the next time step. The vertical velocity $\dot{\eta}$ (a diagnostic output variable from the spectral representation) mapped into a pure pressure vertical coordinate system will be denoted $\omega_{\text {spec }}$ in the following.

The kinematic method implies fundamental problems since the horizontal wind speed in the atmosphere is much higher than the vertical wind speed. As a result, small errors in the horizontal wind may lead to large errors in the vertical wind. Vertical wind fields derived through the continuity equation often show very patchy structures. This phenomenon has been shown to cause excessively dispersive transport (e.g., Schoeberl et al., 2003; Ploeger et al., 2011, although their results are also affected by assimilation effects). 
In the standard configuration of EMAC, an implementation of a flux-form semi-Lagrangian transport scheme (FFSL; Lin and Rood, 1996; Carpenter et al., 1990) is used for the tracer transport. Only the horizontal winds are input parameters for the tracer transport in EMAC. Horizontal tracer mass fluxes are derived using the horizontal wind field. The vertical velocity $\omega_{\text {FFSL }}$ used in the FFSL tracer transport is derived from the continuity equation for the tracer from the horizontal tracer mass fluxes for individual model grid boxes (Lin, 2004; Lauritzen et al., 2011). This vertical velocity $\omega_{\text {FFSL }}$ differs from the vertical velocity $\omega_{\text {spec }}$ deduced from the wind field, since different advection schemes are used for the air-mass density and for trace gases: the spectral advection is used for air-mass density, whereas the grid-pointbased FFSL transport is used for the tracers. Each advection scheme uses its own grid and is internally mass-conservative, but re-mapping of trace gas distributions to the $\eta$-grid can produce inconsistencies. This phenomenon has been investigated in detail by Jöckel et al. (2001).

Within the frame of this work, a diagnostic for vertical velocities was developed and implemented in the EMAC fluxform semi-Lagrangian transport module. The diagnostic for the vertical velocity in the transport scheme is adapted from the Community Atmosphere Model (CAM) finite-volume dynamical core (implemented by $\mathrm{C}$. Chen, and described in Lin, 2004). The internal grid in the FFSL transport module differs from the $\eta$-grid in ECHAM5: it is variable in the vertical dimension and fixed in the horizontal dimensions. This concept is denoted as "vertically Lagrangian" (Lin, 2004) or "floating Lagrangian vertical coordinate" (Lauritzen et al., 2011). In each advection time step, horizontal mass fluxes through the lateral boundaries are calculated for each grid box. Through the advection the mass in each grid box changes and therefore also the thickness of each grid box in a terrain-following pressure-based vertical coordinate system. For the $\omega$-diagnostic the pressure at the layer interfaces before and after the advection is compared. The pressure in one grid box is influenced by the mass in the grid boxes above and by the horizontal mass fluxes into the grid box. This constitutes the vertically Lagrangian character of the advection scheme, since the pressure boundaries of the grid boxes are not fixed. After the advection time step, the new surface pressure based on the new mass distribution in each column of the model grid is calculated. Then, a vertical re-mapping of the trace gas distributions according to the $\eta$-levels defined by the new surface pressure takes place.

The top panels of Fig. 1 present the annual, zonal mean of the vertical velocity from the spectral representation $\bar{\omega}_{\text {spec }}$ and from the transport diagnostic $\bar{\omega}_{\text {FFSL }}$. The differences between $\bar{\omega}_{\text {spec }}$ and $\bar{\omega}_{\text {FFSL }}$ are visualized by showing the absolute values of their absolute and relative differences. The absolute value of absolute difference was derived as $\left|\bar{\omega}_{\text {spec }}-\bar{\omega}_{\text {FFSL }}\right|$, and the absolute value of the relative difference is defined as $\left|\frac{\bar{\omega}_{\text {spec }}-\bar{\omega}_{\mathrm{FFSL}}}{0.5 \cdot\left(\left|\bar{\omega}_{\text {spec }}\right|+\left|\bar{\omega}_{\mathrm{FFSL}}\right|\right)}\right|$.
The comparison of the vertical velocity $\bar{\omega}_{\text {spec }}$ to $\bar{\omega}_{\text {FFSL }}$ reveals that the differences are rather small in most parts of the stratosphere. There are some exceptions of small regions with high relative differences: the minimum in the upwelling pattern at the Equator at $10 \mathrm{hPa}$ is stronger in $\bar{\omega}_{\text {spec }}$, showing even positive values in the annual, zonal mean. Further, the upwelling and downwelling regions are slightly shifted around the contours of $0 \mathrm{hPaday}^{-1}$. Apart from that, the relative differences between $\bar{\omega}_{\text {spec }}$ and $\bar{\omega}_{\text {FFSL }}$ are below $10 \%$ (bottom right panel of Fig. 1). The absolute differences in the annual zonal mean are small throughout the stratosphere (bottom left panel of Fig. 1). In the following analysis the vertical velocity $\omega_{\text {FFSL }}$ obtained from the new diagnostic in the transport scheme is used since this is the actual vertical velocity that causes vertical advection in the FFSL transport scheme. In the following, $\omega_{\text {FFSL }}$ will be denoted $\omega$.

\section{Transformed Eulerian mean}

The calculation of the Eulerian zonal mean $\bar{\omega}$ of the kinematic vertical velocity $\omega$ does not deliver a meaningful representation of the atmospheric diabatic circulation that is relevant for trace gas transport. Planetary waves may induce upwelling and downwelling in the Eulerian zonal mean $\bar{\omega}$ in different latitudes, which is not related to net tracer transport. In this situation, calculating the Eulerian zonal mean of $\omega$ yields zonal mean upwelling and downwelling in different latitudes due to the planetary wave activity, which is not related to net tracer transport (see Fig. 2). A more detailed discussion of this phenomenon is given in, e.g., Brasseur et al. (1999).

The transformed Eulerian mean (TEM) can be used instead of the Eulerian mean to avoid the misleading effects in the zonal mean vertical velocity. The idea of this transformation is to produce a similar picture as if the average vertical velocity was taken along fluid parcel paths. Another idea of this transformation is to find a correct representation of the diabatic vertical velocity in the $p$ space by an appropriate redefining of $v^{*}$ and $w^{*}$ and without changing the continuity equation for $v^{*}$ and $w^{*}$. The TEM mean meridional velocity $\bar{v}^{*}$ and vertical velocity $\bar{w}^{*}$ are defined as follows (e.g., Andrews et al., 1987):

$$
\begin{aligned}
& \bar{v}^{*}=\bar{v}-\frac{1}{\rho_{0}}\left(\frac{\rho_{0} \overline{v^{\prime} \theta^{\prime}}}{\overline{\theta_{z}}}\right)_{z}, \\
& \bar{w}^{*}=\bar{w}+\left(\frac{\overline{v^{\prime} \theta^{\prime}} \cos \phi}{\overline{\theta_{z}}}\right)_{\phi} .
\end{aligned}
$$

Here, $\bar{v}$ denotes the Eulerian mean meridional velocity, $\bar{w}$ the Eulerian mean vertical velocity in log-pressure coordinates, $\overline{v^{\prime} \theta^{\prime}}$ the eddy heat flux, $\bar{\theta}$ the Eulerian mean potential temperature, subscript $z$ denotes the partial derivative in the vertical $\left(\frac{\partial}{\partial z}\right)$, and $\phi$ latitude. $\rho_{0}(z) \equiv \rho_{0} \cdot e^{-z / H}$ is the basic mass density with $\rho_{0}$ denoting the mass density at the reference surface pressure $p_{0}$. The log-pressure height $z$ is derived 

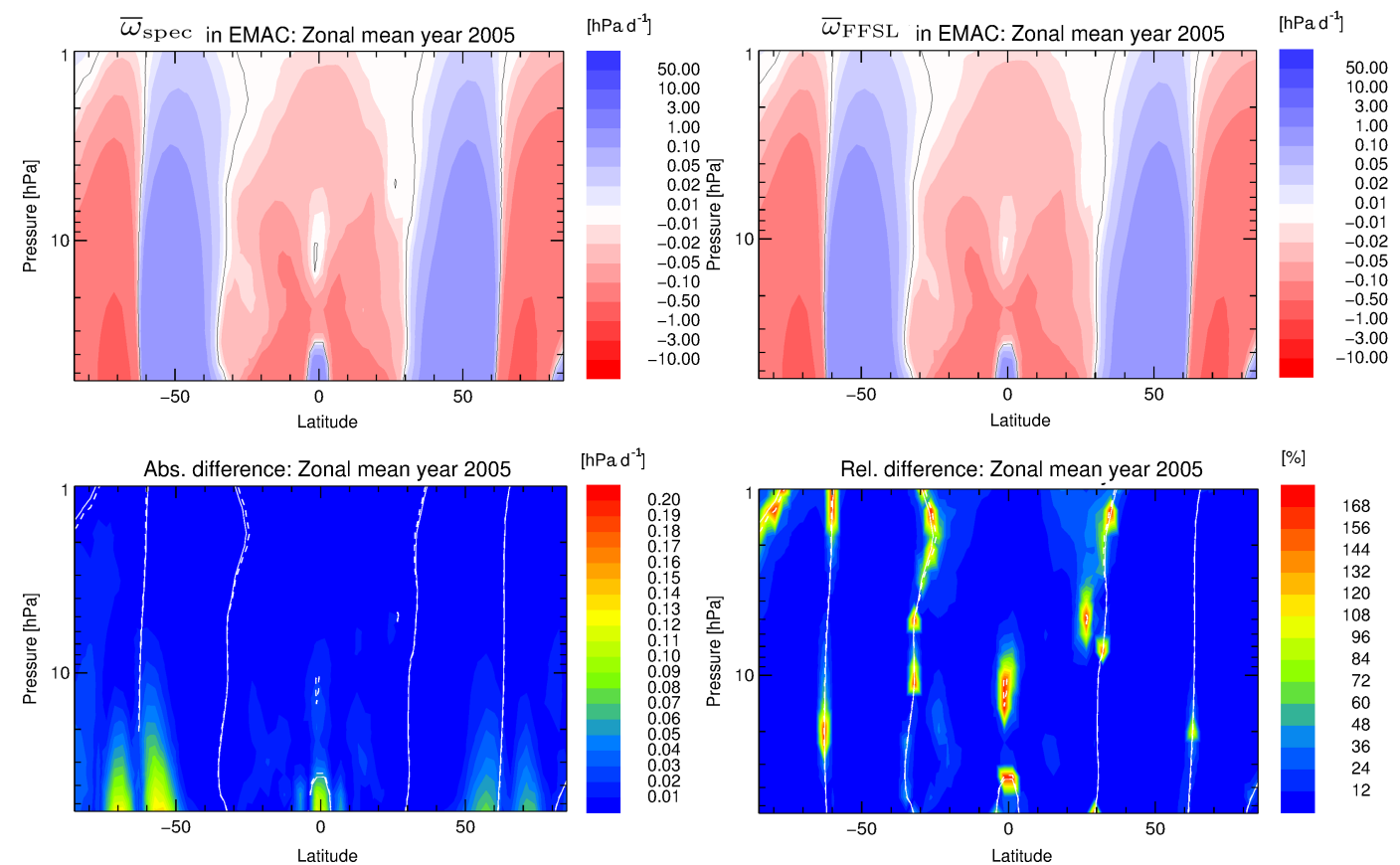

Figure 1. Annual, zonal mean $\bar{\omega}_{\text {spec }}$ (top left panel) and $\bar{\omega}_{\text {FFSL }}$ (top right panel) (hPaday ${ }^{-1}$ ) for the year 2005 . Here, black solid lines show the $0 \mathrm{hPaday}^{-1}$ contour of the respective vertical velocities. The bottom panels show absolute value of absolute difference (hPa day ${ }^{-1}$ ) (left) and relative difference (right) between $\bar{\omega}_{\text {spec }}$ and $\bar{\omega}_{\text {FFSL }}$. White dashed and solid lines in the bottom panels display the $0 \mathrm{hPa}$ day ${ }^{-1}$ contour of $\bar{\omega}_{\text {spec }}$ and $\bar{\omega}_{\text {FFSL }}$, respectively.

from pressure $p$ through

$z=-H \cdot \ln \frac{p}{p_{0}}$.

In this study, surface pressure $p_{0}$ and scale height $H$ were set to $1000 \mathrm{hPa}$ and $7 \mathrm{~km}$, respectively. The circulation described by $\bar{v}^{*}$ and $\bar{w}^{*}$ is called the residual mean mass circulation.

Figure 2 shows the zonal mean vertical velocity $\bar{w}$ and the TEM vertical velocity $\bar{w}^{*}$ from EMAC for the year 2005 . The zonal mean vertical velocity $\bar{w}$ in the top panel of Fig. 2 features a pronounced downwelling in the 40 to $60^{\circ}$ latitude region and an upwelling in the polar regions from $60^{\circ}$ latitude to the poles in both hemispheres. This pattern is due to eddy flux divergences and the zonal mean $\bar{w}$ thus gives a very misleading picture. The TEM vertical velocity $\bar{w}^{*}$ in the bottom panel of Fig. 2 represents the relevant circulation for zonal mean tracer transport. Here, the circulation shows downwelling throughout the entire extratropical stratosphere in the annual mean, as expected.

\subsection{Diabatic vertical velocity}

In EMAC-CLaMS potential temperature is used as the vertical coordinate in the stratosphere (Hoppe, 2014). The vertical velocity $\dot{\theta}$ in this representation is derived from the diabatic heating rate $Q$ :

$\dot{\theta}=Q \frac{\theta}{T}$.

Here, diabatic heating rate means $Q=J / c_{p}$, where $J$ is the diabatic heating rate per unit mass and $c_{p}$ the specific heat capacity at constant pressure. Transport across isentropic surfaces can take place only through diabatic heating. The diabatic heating rate $Q$ is the sum of radiative heating $Q_{\mathrm{rad}}$, heating from diffusion and turbulent mixing $Q_{\text {diff }}$ and heating from latent heat release $Q_{\text {lat }}$ :

$Q=Q_{\text {rad }}+Q_{\text {diff }}+Q_{\text {lat }}$.

The radiative heating $Q_{\text {rad }}$ is the dominant term in the stratosphere, while in the tropopause region the latent heat release is also of importance (Ploeger et al., 2010). The contributions of the different terms to the diabatic heating rate in the ERA-Interim reanalysis were also investigated by Fueglistaler et al. (2009) and Wright and Fueglistaler (2013).

A diagnostic tool to capture the diabatic heating from the different process parametrizations in EMAC was implemented during this work. A slightly modified version of the tendency diagnostic of the ECHAM6 model (Stevens et al., 2013) was used for this task (S. Rast, personal communication, 2013). The diagnostic reads the temperature before and after processes that cause diabatic heating and calculates 

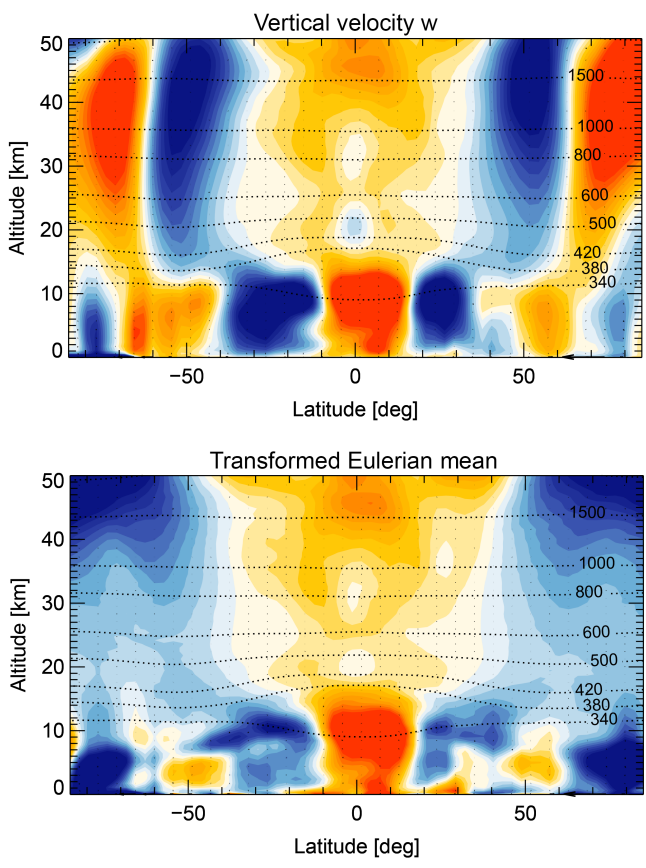

$-0.0120-0.0016-0.0010-0.00040 .0002 \quad 0.0008 \quad 0.0014 \quad 0.0020$

Figure 2. Annual, zonal mean vertical velocity $\bar{w}\left(\mathrm{~ms}^{-1}\right)$ (top panel) and transformed Eulerian mean (TEM) vertical velocity $\bar{w}^{*}$ $\left(\mathrm{m} \mathrm{s}^{-1}\right)$ (bottom panel) from EMAC for the year 2005. Dotted lines display potential temperature levels $(\mathrm{K})$. The vertical axis displays log-pressure height $(\mathrm{km})$, calculated from Eq. (4).

temperature tendencies $\Delta T\left(\mathrm{~K} \mathrm{~s}^{-1}\right)$. Let $\Delta T^{(i)}$ be the temperature tendency caused by process $i$. If temperature $T$ at time $t$ is changed by $n$ different processes in the model time step $\Delta t$, then the temperature in the next time step $T(t+\Delta t)$ reads

$T(t+\Delta t)=T(t)+\sum_{i=1}^{n} \Delta T^{(i)}(t) \Delta t$

The temperature tendencies $\Delta T^{(i)}$ from all processes that cause diabatic heating are added up. The vertical velocity $\dot{\theta}$ is then determined by Eq. (5). In EMAC, the parametrizations for radiation, convection, clouds, vertical diffusion, and gravity wave drag contribute to the total diabatic heating rate $Q$. Most of the processes mentioned above cannot be resolved by the coarse model grid and have to be parametrized. However, subgrid parametrizations always imply a certain degree of inaccuracy. Different parametrizations of the same process deliver different results. For example the choice of the convection scheme influences the diabatic vertical velocity in the tropical tropopause region (TTL; see Appendix A). For this study, the parametrizations of subgrid processes were set to the standard EMAC configuration (see Table 1).
Table 1. Parametrizations in EMAC.

\begin{tabular}{|c|c|}
\hline Process & Scheme \\
\hline Clouds & $\begin{array}{l}\text { ECHAM5 cloud scheme } \\
\text { (Röckner et al., 2006, and references therein) }\end{array}$ \\
\hline Convection & $\begin{array}{l}\text { Tiedtke convection with Nordeng closure } \\
\text { (Tiedtke, 1989; Nordeng, 1994) }\end{array}$ \\
\hline Gravity waves & Hines scheme (Hines, 1997) \\
\hline Radiation & $\begin{array}{l}\text { ECHAM5 radiation scheme* (Jöckel et al., 2006, } \\
\text { Röckner et al., 2006, and references therein) }\end{array}$ \\
\hline
\end{tabular}

\section{Vertical velocity climatologies}

This section presents zonal mean climatologies of diabatic and kinematic vertical velocity in EMAC and analyzes the differences between these vertical velocity representations. These zonal mean climatologies for $\bar{\omega}$ were produced by interpolating the model data (mean values over the model time step of $15 \mathrm{~min}$ ) onto a regular vertical grid in $\theta$ coordinates and calculating the zonal mean value over the 10-year simulation. For this comparison, both velocities have been converted to comparable quantities (namely, $\omega=\frac{\mathrm{D} p}{\mathrm{D} t}$ ). The kinematic vertical velocity $\bar{w}^{*}$ (defined in the log-pressure coordinate system and calculated in the TEM formalism) has been converted to $\bar{\omega}^{*}$ in pressure coordinates using the definition of the log-pressure height (Eq. 4):

$\bar{\omega}^{*}=-\frac{\bar{w}^{*} \cdot p}{H}$.

Equation (8) is only valid for model layers of constant pressure $p$. The EMAC hybrid model layers are defined such that above about $55 \mathrm{hPa}$ the pressure at the model layers is constant.

The diabatic vertical velocity $\dot{\theta}$ was converted to the respective velocity $\bar{\omega}_{\theta}$ in pressure coordinates by using the definition of the total derivative of $\theta$ in spherical coordinates:

$\frac{\mathrm{D} \theta}{\mathrm{D} t}=\frac{\partial \theta}{\partial t}+\frac{1}{r_{\mathrm{E}}} u \frac{\partial \theta}{\partial \lambda}+\frac{1}{r_{\mathrm{E}} \cos \phi} v \frac{\partial \theta}{\partial \phi}+\omega \frac{\partial \theta}{\partial p}$.

Here, $\lambda, \phi$, and $r_{\mathrm{E}}$ denote longitude, latitude, and the radius of the Earth, respectively. Solving Eq. (9) for $\omega$ leads to

$\omega_{\theta}=\frac{-\frac{\partial \theta}{\partial t}-\frac{1}{r_{\mathrm{E}}} u \frac{\partial \theta}{\partial \lambda}-\frac{1}{r_{\mathrm{E}} \cos \phi} v \frac{\partial \theta}{\partial \phi}+\dot{\theta}}{\frac{\partial \theta}{\partial p}}$.

The robustness of this transformation has been checked by first applying Eq. (10) and then using the inverse transformation to convert $\omega_{\theta}$ to $\dot{\theta}_{\text {test }}$. The differences between the original $\dot{\theta}$ and $\dot{\theta}_{\text {test }}$ are found to be smaller than $10^{-6} \mathrm{~K}$ (not shown). Wohltmann and Rex (2008) used this transformation (Eq. 10) in a similar way. 

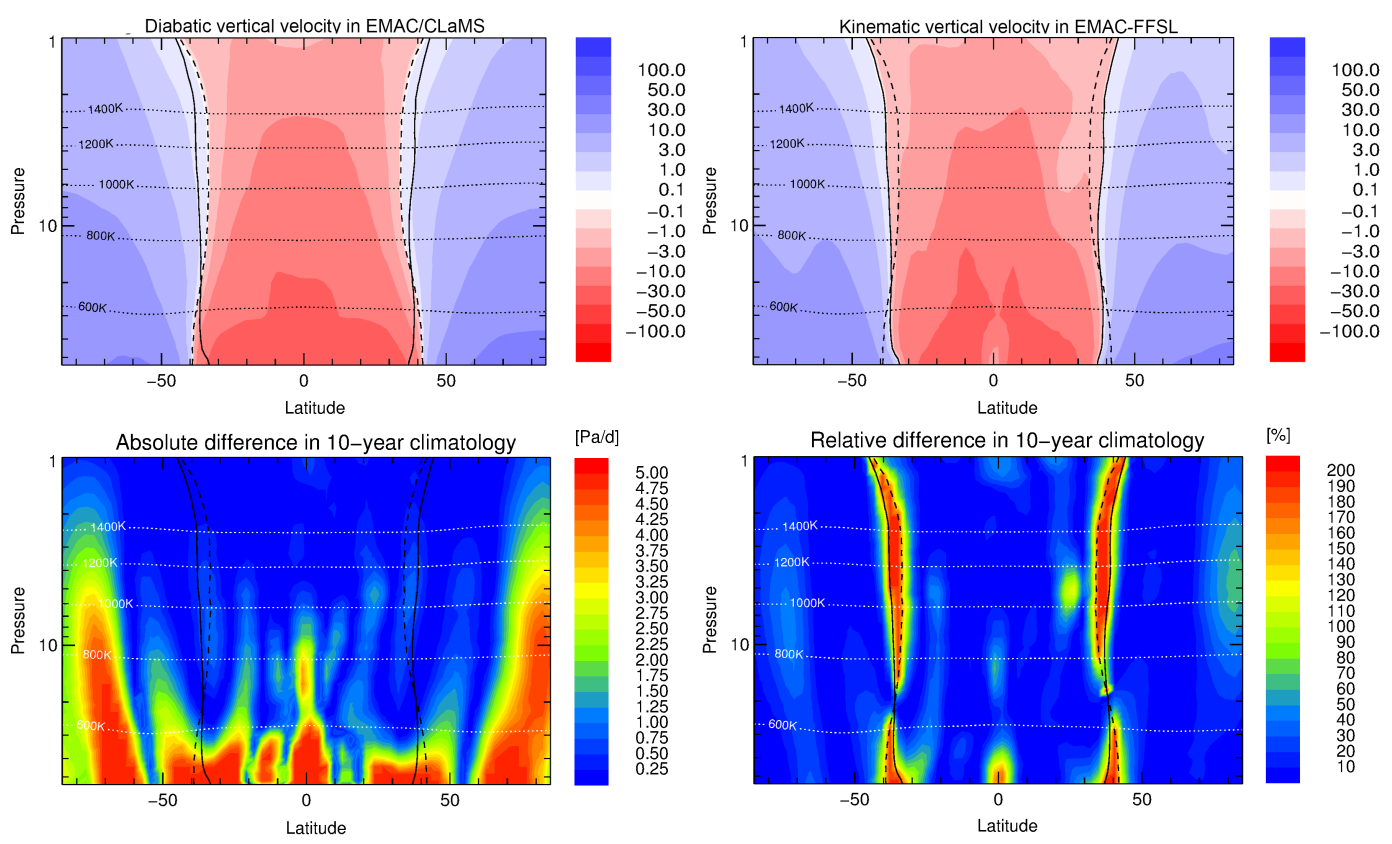

Figure 3. Diabatic vertical velocity $\bar{\omega}_{\theta}$ from diabatic heating rates (top left panel) and transformed Eulerian mean (TEM) vertical velocity $\bar{\omega}^{*}$ (top right panel) from 10-year EMAC climatology $\left(\mathrm{Pa} \mathrm{day}^{-1}\right)$. The bottom panels show absolute value of absolute difference $\left(\mathrm{Pa}\right.$ day ${ }^{-1}$ ) (left) and relative difference (right) between $\bar{\omega}_{\theta}$ and $\bar{\omega}^{*}$. Dashed and solid black contours indicate the turnaround latitudes of $\bar{\omega}_{\theta}$ and $\bar{\omega}^{*}$, respectively. Dotted lines display levels of constant potential temperature $\theta$.

This section presents a comparison of the annual, zonal mean of the diabatic vertical velocity $\bar{\omega}_{\theta}$ calculated from Eq. (10) and the kinematic vertical velocity $\bar{\omega}^{*}$ according to the TEM formulation in the 10-year EMAC simulation, whereas monthly climatologies are presented in Appendix B. Figure 4 presents respective climatologies for the seasons December to February and June to August.

Figure 3 shows that the 10-year mean of both vertical velocity representations exhibits continuous upwelling at low latitudes and continuous downwelling at higher latitudes and in the polar regions.

The relative and absolute differences between $\bar{\omega}_{\theta}$ and $\bar{\omega}^{*}$ are also presented in Fig. 3. There are notable differences in the shape of the upwelling region (tropical pipe): the turnaround latitudes in both hemispheres of $\bar{\omega}^{*}$ are nearly constant with height up to $2 \mathrm{hPa}$, so that the tropical pipe in the kinematic vertical velocity field is almost straight. In contrast, the tropical pipe of the diabatic vertical velocity $\bar{\omega}_{\theta}$ has a different shape. It is wider than the upwelling region of $\bar{\omega}^{*}$ up to $20 \mathrm{hPa}$ and narrower at higher altitudes. In Fig. 3 the turnaround latitudes of $\bar{\omega}_{\theta}$ and $\bar{\omega}^{*}$ can directly be compared to each other. At $2 \mathrm{hPa}$ the turnaround latitudes of the diabatic velocity are located at $35^{\circ}$ latitude while they are found at $40^{\circ}$ latitude in the kinematic velocity field. The different shape of the tropical upwelling region causes the largest relative differences between $\bar{\omega}_{\theta}$ and $\bar{\omega}^{*}$ (bottom right panel of Fig. 3), though the absolute differences around the turnaround latitudes above $600 \mathrm{~K}$ are small (bottom left panel of Fig. 3).

The upwelling at around $50 \mathrm{hPa}$ extends to higher latitudes in $\bar{\omega}_{\theta}$ in both hemispheres, i.e., from $40^{\circ} \mathrm{S}$ to $42^{\circ} \mathrm{N}$ in $\bar{\omega}_{\theta}$ compared to $35^{\circ} \mathrm{S}$ to $37^{\circ} \mathrm{N}$ in $\bar{\omega}^{*}$. The upwelling is stronger in the diabatic vertical velocity field in the latitude range between 30 and $40^{\circ}$ in both hemispheres.

In general, the circulation pattern is more uniform using diabatic vertical velocities. The kinematic vertical velocities exhibit more structures, even in the 10-year zonal mean, than the diabatic vertical velocity. In particular, the kinematic vertical velocity shows an equatorial minimum, a minimum in downwelling at $75^{\circ} \mathrm{S}$, and a minimum in upwelling at $30^{\circ} \mathrm{N}$ between 1000 and $1200 \mathrm{~K}$. The minimum at $55 \mathrm{hPa}$ or $500 \mathrm{~K}$ over the Equator is also present in the ERA-Interim reanalysis (Seviour et al., 2012) and in other climate models (Butchart et al., 2006) using kinematic vertical velocity. At higher altitudes, directly at the Equator the mean kinematic vertical velocity $\bar{\omega}^{*}$ is lower than at $10^{\circ}$ latitude. This is visible, e.g., in the $-3 \mathrm{Paday}^{-1}$ contour of $\bar{\omega}^{*}$ at $1300 \mathrm{~K}$ over the Equator in the top right panel of Fig. 3. The equatorial minimum is not seen in the 10-year mean in the diabatic vertical velocity pattern. The diabatic vertical velocity shows maximum values around $0^{\circ}$ latitude and therefore stronger upwelling above the Equator than the kinematic vertical velocity. The differences due to the minima of $\bar{\omega}^{*}$ are clearly visible in the absolute and relative difference patterns (bottom panels of Fig. 3). The noisier structure of $\bar{\omega}^{*}$ compared 

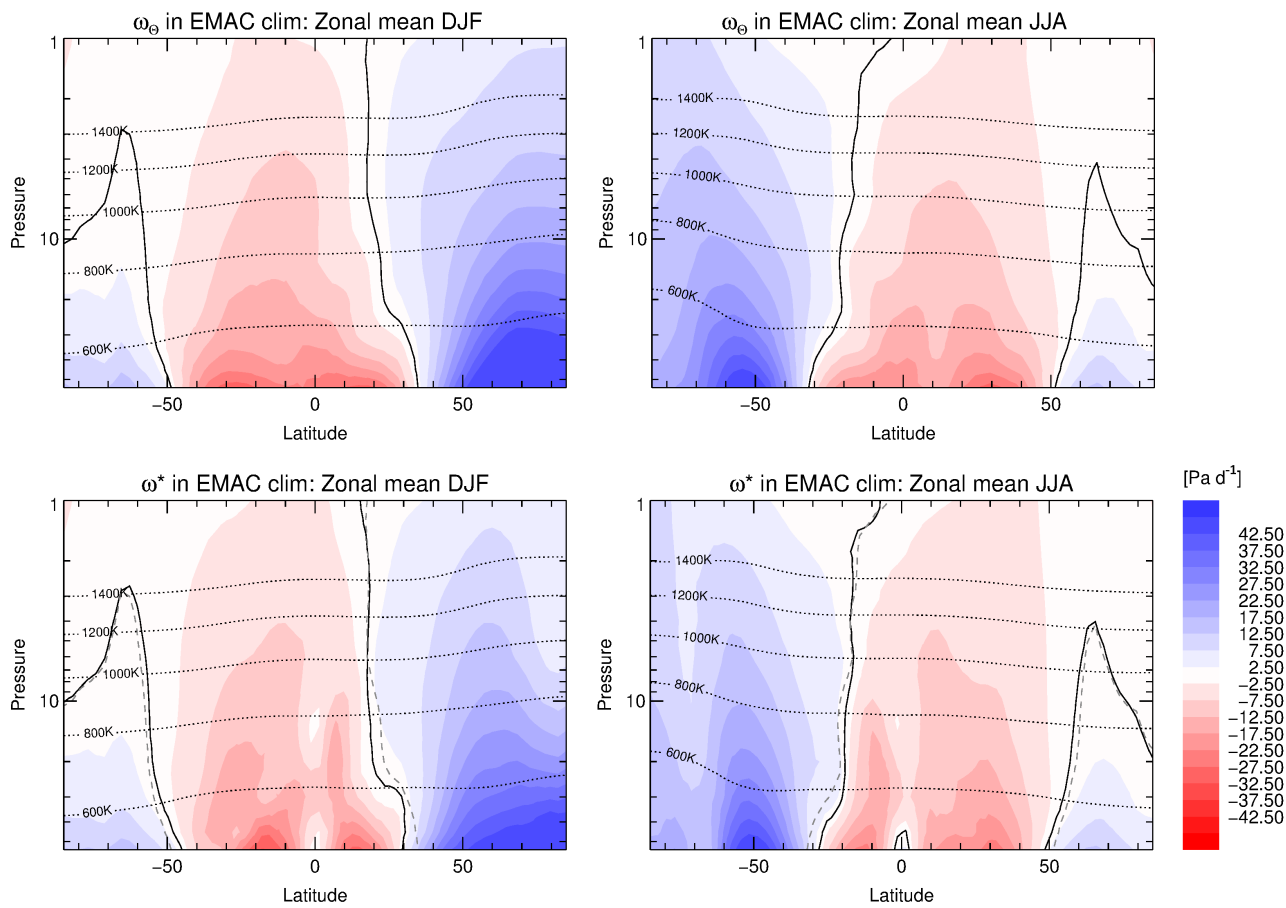

Figure 4. Diabatic vertical velocity $\bar{\omega}_{\theta}$ from diabatic heating rates (top panels) and transformed Eulerian mean (TEM) vertical velocity $\bar{\omega}^{*}$ (bottom panels) from 10-year EMAC climatology $\left(\mathrm{Paday}^{-1}\right.$ ) for the seasons December to January (left panels) and June to August (right panels). Solid black contours indicate the turnaround latitudes of $\bar{\omega}_{\theta}$ (top panels) and $\bar{\omega}^{*}$ (bottom panels). Dashed contours in the bottom panels display the respective turnaround latitudes of $\bar{\omega}_{\theta}$.

to $\bar{\omega}_{\theta}$ is more pronounced in monthly climatologies (see Appendix B).

Above $15 \mathrm{hPa}$, the tropical pipe is wider in $\bar{\omega}^{*}$ than in $\bar{\omega}_{\theta}$ but the region of the strongest upwelling is narrower. This is indicated by the $-3 \mathrm{Paday}^{-1}$ contour in the top panels of Fig. 3. While this contour is nearly symmetric in the diabatic vertical velocity field, it has a maximum at $10^{\circ} \mathrm{N}$ in the kinematic representation. At lower altitudes at about $15 \mathrm{hPa}$, both velocity patterns show a maximum upwelling in the Southern Hemisphere (SH). This is a realistic representation of the diabatic circulation, since the maximum upwelling is observed during Northern Hemisphere $(\mathrm{NH})$ winter, where strong wave activity is observed in the NH (Randel et al., 2008). Downwelling in the polar vortex regions is stronger using diabatic vertical velocity. The absolute differences between $\bar{\omega}_{\theta}$ and $\bar{\omega}^{*}$ are large in the polar regions. This is visible in Fig. 3 since the $10 \mathrm{Paday}^{-1}$ contour is located at higher altitudes in $\bar{\omega}_{\theta}$ in the region from $60^{\circ}$ latitude to the pole in both hemispheres. Additionally, the seasonal plots in Fig. 4 display the differences in downwelling in the polar vortex regions.

One important difference between the kinematic and the diabatic vertical velocity representation is illustrated in the left panel of Fig. 5. This contour plot shows selected isolines of zonal mean upwelling velocities of the two transport schemes for February. This figure reveals that the upwelling in $\mathrm{NH}$ winter (here: February) in the $\mathrm{SH}$ tropics is stronger using diabatic vertical velocities than when using kinematic vertical velocities. This difference in the vertical velocities has an impact on the simulated trace gas and age of air patterns (Sect. 4).

The contour plot in the right panel of Fig. 5 shows the corresponding isolines of zonal mean upwelling velocities for July. It is clearly visible that the region of the strongest upwelling in $\bar{\omega}_{\theta}$ is shifted southwards compared to the upwelling region of $\bar{\omega}^{*}$ above $15 \mathrm{hPa}$. The $-5 \mathrm{Paday}^{-1}$ isoline reveals that the maximum upwelling region in the diabatic vertical velocity field is shifted southwards by about $5^{\circ} \mathrm{com}-$ pared to the kinematic vertical velocity. The $-12 \mathrm{Paday}^{-1}$ isoline of the diabatic vertical velocity also exhibits a southward shift in the $\mathrm{NH}$ upwelling region. This shift has a large impact on trace gas distributions, as will be shown in the following.

To summarize, the kinematic and the diabatic vertical velocities in EMAC show roughly similar seasonal variations. The main differences between these two vertical velocity representations are

- a noisier kinematic vertical velocity pattern

- higher diabatic vertical velocities in the upwelling regions in the inner tropics and in the downwelling regions in the polar vortex 

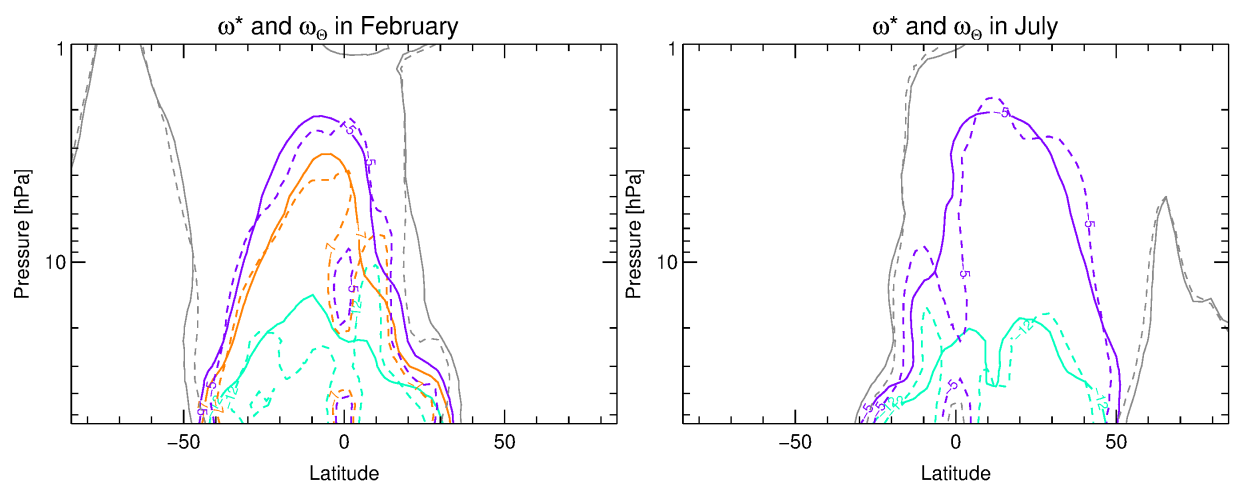

Figure 5. Kinematic vertical velocity $\left(\bar{\omega}^{*}\right.$, dashed lines) and diabatic vertical velocity $\left(\bar{\omega}_{\theta}\right.$, solid lines) for February (left panel) and July

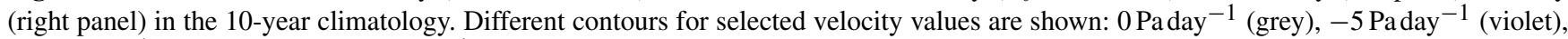
$-7 \mathrm{Paday}^{-1}$ (orange), and $-12 \mathrm{Paday}^{-1}$ (turquoise).

- a southward shift of maximum upwelling in the diabatic vertical velocity in $\mathrm{NH}$ summer

- a narrower upwelling region in the zonal mean diabatic vertical velocity.

\section{Impact on mean age of air distributions}

This section shows mean age of air climatologies from a 10year time-slice simulation with the coupled EMAC-CLaMS model. The setup is described in detail by Hoppe et al. (2014). In this simulation, two transport schemes using different vertical velocities were applied with two similar tracer sets including a mean age of air tracer (for details see Pommrich et al., 2014), implemented as a passive tracer with a linearly increasing lower boundary condition ("clock-tracer"; Hall and Plumb, 1994). The mean age at a certain position in the atmosphere is derived from the difference between the local tracer value and the current value at the surface. Tracer distributions calculated with the Lagrangian CLaMS transport scheme (with diabatic vertical velocity) are compared to tracer fields derived from the FFSL transport (with kinematic vertical velocity) in EMAC. The transport with the fullLagrangian transport scheme will be referred to as "EMACCLaMS" in the following, and the one using the FFSL transport will be denoted "EMAC-FFSL".

Figure 6 shows zonal mean age of air climatologies for EMAC-FFSL and EMAC-CLaMS. Both age of air distributions are consistent with the known features of the stratospheric Brewer-Dobson circulation. Young air masses are present at low latitudes due to upwelling in the tropical pipe. At high latitudes, the air is older with age of air values higher than 4.75 years in the annual, zonal mean.

In addition, the results of an analysis of the residual circulation transit times (RCTTs) analysis (Garny et al., 2014; Ploeger et al., 2015) are presented. This method determines the age of air that would be present if there was only residual circulation without any eddy mixing present in the at- mosphere. The kinematic and the diabatic vertical velocities serve as input for the RCTT analysis and the results are shown in Fig. 7. Residual circulation trajectories for kinematic vertical velocity were calculated in pressure coordinates, where diabatic trajectories were calculated in potential temperature coordinates using the isentropic mass-weighted residual circulation (e.g., Ploeger et al., 2015). Evidently, the faster residual circulation of the diabatic vertical velocities in EMAC-CLaMS lead to lower RCTTs in most parts of the stratosphere. Also, the transition to higher RCTTs happens at lower latitudes using EMAC-CLAMS.

In the following, the differences in the age of air patterns of EMAC-CLaMS and EMAC-FFSL (bottom panel of Fig. 6) will be discussed. Several differences in the age of air distributions are consistent with the vertical velocity differences that are discussed in the previous section. By showing the age of air climatologies and results from the RCTT analysis, this section discusses to what extent mean age of air distributions allow for conclusions on the residual circulation to be drawn. Similarities in the difference patterns of age of air and RCTTs (shown in the bottom panels of Figs. 6 and 7, respectively) indicate that differences in the age of air pattern are due to differences in the residual circulation, whereas different patterns show that the differences in age of air are due to mixing effects. Note that a difference between mean age and RCTT is indicative for both large-scale eddy mixing and small-scale diffusion effects (e.g., Garny et al., 2014).

There are notable differences in the age of air pattern between EMAC-CLaMS and EMAC-FFSL (bottom panel of Fig. 6). The most obvious pattern in the age of air differences is the hemispheric age difference at altitudes from 50 to $5 \mathrm{hPa}$. Here, the usage of EMAC-CLaMS results in younger air in the SH and older air in the NH compared to EMACFFSL (see also Hoppe, 2014). The RCTT analysis shows no analogous hemispheric pattern and, therefore, the additional effects of mixing are the main cause for the hemispheric pattern in mean age of air. The northward shift of the maximum upwelling in the kinematic vertical velocity field of EMAC- 

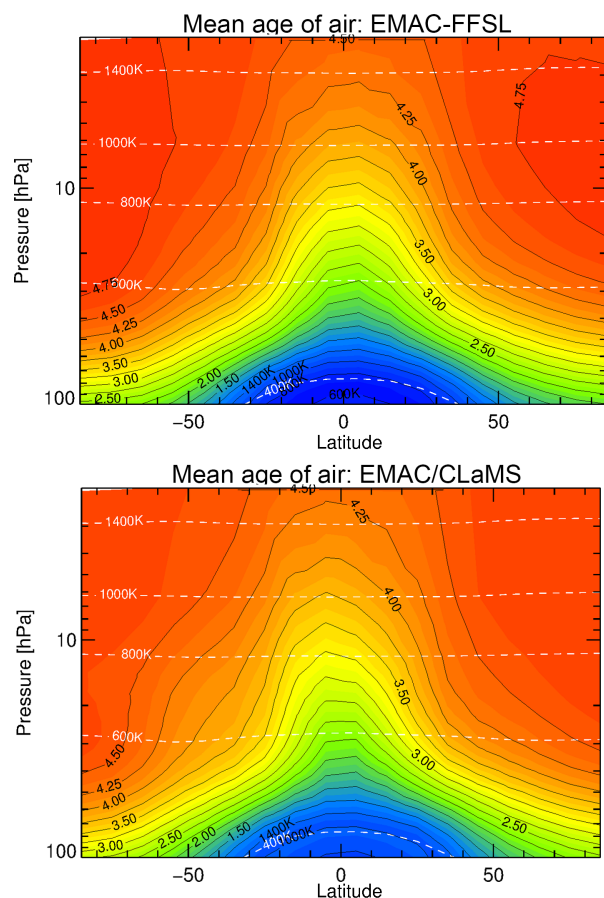

Difference: EMAC/CLaMS - EMAC-FFSL

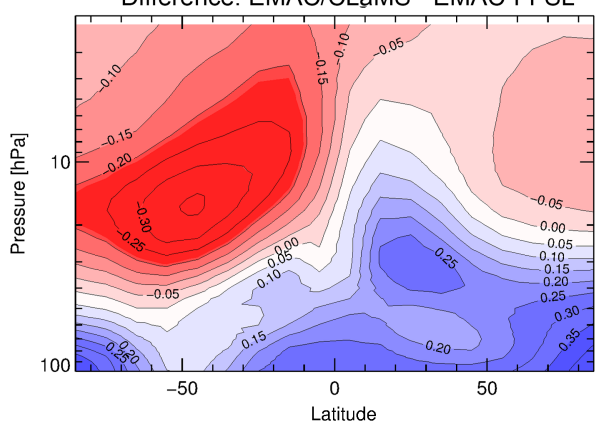

Figure 6. Annual, zonal mean age of air from 10-year climatologies (years) for EMAC-FFSL (top panel) and EMAC-CLaMS (middle panel). Dashed lines show levels of constant potential temperature $\theta$. Absolute differences in age of air (EMAC-CLaMS - EMACFFSL) (years) are shown in the bottom panel. Blue colors indicate younger air in EMAC-FFSL, while red colors indicate younger air in EMAC-CLaMS.

FFSL compared to the diabatic upwelling of EMAC-CLaMS (right panel of Fig. 5) is most pronounced at altitudes above $15 \mathrm{hPa}$. Thus, related differences in the vertical velocities only have a minor contribution limited to the upper part of the difference pattern at $10 \mathrm{hPa}$ from $20^{\circ} \mathrm{S}$ to $20^{\circ} \mathrm{N}$, where the absolute values of the RCTT differences are higher in the $\mathrm{SH}$ than in the NH.

In the inner tropics from about $10^{\circ} \mathrm{S}$ to $10^{\circ} \mathrm{N}$ latitude above $50 \mathrm{hPa}$, the mean age of air is younger in EMACCLaMS, as expected from higher diabatic vertical velocities in this region (left panel of Fig. 5). This is confirmed in the RCTT analysis, since here, the RCTTs are clearly lower in EMAC-CLaMS.
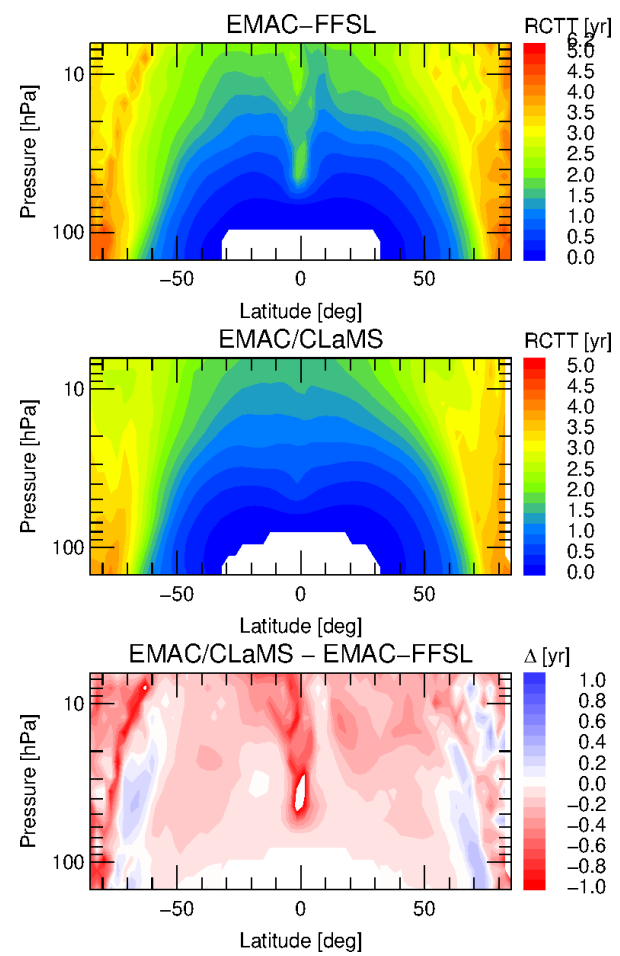

Figure 7. Residual circulation transit times (RCTTs) for EMACFFSL (top panel) and EMAC-CLaMS (middle panel) (years). Absolute differences in RCTTs (EMAC-CLaMS - EMAC-FFSL) (years) are shown in the bottom panel. Blue colors indicate lower RCTTs in EMAC-FFSL, while red colors indicate lower RCTTs in EMAC-CLaMS.

The age of air is younger in EMAC-FFSL in the extratropical lowest part of the stratosphere (below $50 \mathrm{hPa}$ ). This effect is likely due to a lower permeability of the tropopause in EMAC-CLaMS causing reduced cross-tropopause diffusion for Lagrangian transport. The RCTT analysis shows that this is a result of mixing, since this pattern is not visible in the RCTT differences between EMAC-CLaMS and EMACFFSL.

Thus, there are two distinct features of the transport schemes (EMAC-FFSL and EMAC-CLaMS) that are responsible for the different distributions of mean age of air. The first feature is the use of different vertical velocities due to different vertical coordinates. Second, the different transport schemes lead to diverse mixing properties of transport (e.g., Garny et al., 2014; Ploeger et al., 2015). Only by considering both aspects, all differences in the global, zonal mean age of air distributions of EMAC-FFSL and EMACCLaMS can be explained. The vertical velocity obtained by the method presented in this paper is valuable for further analyses like the RCTT diagnostic, which is able to determine the relative contributions of vertical velocity (residual circulation) and additional mixing processes on mean age of air. 


\section{Conclusions}

This work presents climatologies of kinematic and diabatic vertical velocities from the chemistry climate model EMACCLaMS. The diagnostics to obtain the vertical velocities from this model are described in detail.

Annual and monthly zonal mean climatologies of kinematic and diabatic vertical velocity are presented. An analysis of these climatologies reveals several differences between kinematic and diabatic vertical velocity in EMAC: the kinematic vertical velocity field is more noisy and has several minima in the zonal mean distribution. In contrast, the diabatic vertical velocity field is more uniform, and shows higher vertical wind speed in the upwelling region in the inner tropical pipe and the downwelling regions in the polar vortex. There is a shift of the region of maximum upwelling, in particular in boreal summer: the upwelling region is shifted southwards in the diabatic vertical velocity field compared to the kinematic vertical velocity.
The vertical velocity fields have an impact on age of air and trace gas distributions. This work presents a comparison of age of air distributions that were computed using different transport schemes, and using kinematic vertical velocity or diabatic vertical velocity. In some regions, like the upwelling region in the inner tropics, there is a clear correlation between vertical velocity and age of air. However, globally, mixing processes in the atmosphere are equally important. In this study we found that the hemispheric difference pattern in mean age of air is mainly due to mixing effects. Thus, to compare the residual circulation in different CCMs, a comparison of age of air or trace gas distributions alone is not sufficient. Instead, the vertical velocity must be diagnosed explicitly to obtain information about the residual circulation in the model. 


\section{Appendix A: Convection parametrizations in diabatic vertical velocity}

To investigate the impact of the convection scheme on vertical velocity, simulations were run with different convection schemes for the year 2005. Figure A1 shows the annual zonal mean of the diabatic vertical velocity in EMAC using three different convection schemes, namely, the standard Tiedtke convection scheme (Tiedtke, 1989; Nordeng, 1994), the operational ECMWF convection scheme (Tiedtke, 1989; Bechtold et al., 2004), and the Zhang-McFarlaneHack (ZFH) convection scheme (Hack, 1994; Zhang and McFarlane, 1995). The figure focuses on the region of the tropical tropopause layer (TTL), which is the crucial region for tropospheric air entering the stratosphere. In this region, the vertical velocity is small compared to other regions of the atmosphere and small differences in upwelling have a large impact on the trace gas transport. All other process parametrizations are unchanged. The ECMWF convection leads to the strongest vertical upwelling in the tropics. The ZFH convection shows the weakest upwelling, and the strength of upwelling in the Tiedtke convection scheme is in between the other two convection schemes. Another difference is found in the strength of the transport barrier at the level of zero radiative heating at about $350 \mathrm{~K}$. The Tiedtke and the ECMWF convection scheme lead to a strong barrier to vertical transport with an extensive layer of negative vertical velocities in the annual mean at approximately $350 \mathrm{~K}$. However, this transport barrier is not present throughout the year and thus upward transport into the stratosphere is not completely inhibited. In some seasons, there are regions with positive vertical velocities at this altitude. Further, in a model simulation, there will still be an exchange of tropospheric and stratospheric air through vertical numerical diffusion, if the layer of negative vertical velocities is sufficiently thin. The $\mathrm{ZFH}$ convection does not show the layer with negative vertical velocities extending throughout the tropics in the annual mean. Here, at $5^{\circ} \mathrm{S}$ and $5-10^{\circ} \mathrm{N}$ the annual mean has small positive values of the vertical velocity. Overall, there are clear differences in the TTL region using different convection schemes, with the Tiedtke and ECMWF convection showing stronger upwelling between 300 and $340 \mathrm{~K}$ and a more pronounced transport barrier at the level of zero radiative heating $(\approx 350 \mathrm{~K})$ than the $\mathrm{ZFH}$ convection. The influence of choice of convection scheme in EMAC on the hydrological cycle is analyzed in detail in Tost et al. (2006). The authors find that the tested convection schemes show varying skill levels for different aspects of the simulation. Thus, they do not give a recommendation for a specific convection scheme. In the present work, the Tiedtke parametrization is used.

The diabatic vertical velocity using the Tiedtke parametrization has been compared to the respective diabatic vertical velocities that result from the ECMWF and ZFH convection schemes. Note that the convection experiments shown here are only run for 1 year for demonstration purposes, and thus do not ensure a statistically robust comparison. Figure A2 shows the absolute value of absolute differences of diabatic vertical velocity in a way that the plot can be compared to the differences in Fig. 3, bottom left panel. The comparison shows that the differences between the diabatic vertical velocities resulting from Tiedtke and ECMWF convection are in the same order of magnitude as the differences between diabatic and kinematic vertical velocity in the lower part of the tropical pipe between $30^{\circ} \mathrm{S}$ and $30^{\circ} \mathrm{N}$ at $50 \mathrm{hPa}$. At the Equator, the difference pattern reaches up to $10 \mathrm{hPa}$. The differences between the diabatic vertical velocities resulting from Tiedtke and ZFH are smaller and distributed over the latitudes. 

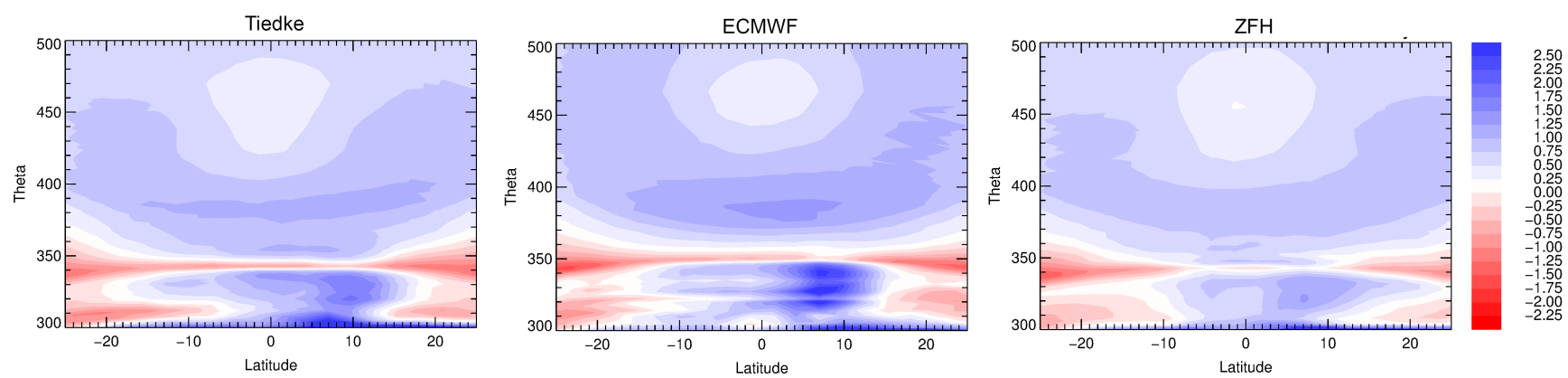

Figure A1. Annual, zonal mean of diabatic vertical velocity $\dot{\theta}\left(\mathrm{Kday}^{-1}\right)$ in EMAC for the year 2005 using the standard Tiedtke convection scheme (left panel), the ECMWF convection scheme (middle panel), and the ZFH convection scheme (right panel).
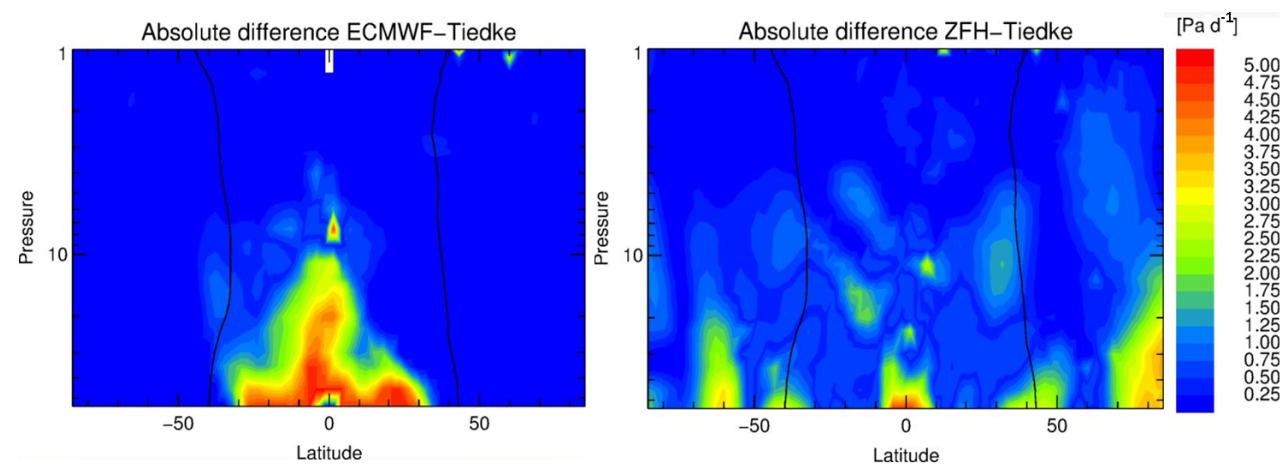

Figure A2. Absolute value of absolute differences in diabatic vertical velocity ( $\mathrm{Pa} \mathrm{day}^{-1}$ ) between the standard Tiedtke convection scheme and the ECMWF convection scheme (left panel) and between the standard Tiedtke convection scheme and the ZFH convection scheme (right panel) in EMAC for the year 2005. 


\section{Appendix B: Monthly climatologies of diabatic and kinematic vertical velocity}

This section presents zonal mean diabatic vertical velocities $\bar{\omega}_{\theta}$ and kinematic vertical velocities $\bar{\omega}^{*}$ from the 10 -year simulation climatology for each month (see Figs. B1 and B2). The seasonal cycle in the stratospheric circulation is clearly visible in both vertical velocity representations. The most remarkable difference between the two transport schemes is the more uniform upwelling and downwelling of $\bar{\omega}_{\theta}$. This feature is more clearly visible in the monthly mean than in the annual mean, since the $\bar{\omega}^{*}$ is much more noisy in the monthly mean compared to the annual mean even when considering a 10-year climatology. The kinematic vertical velocity $\bar{\omega}^{*}$ exhibits several minima in the upwelling and downwelling regions which do not appear in the diabatic $\bar{\omega}_{\theta}$. The most pronounced minimum in the upwelling of $\bar{\omega}^{*}$ is located at the Equator at $55 \mathrm{hPa}$. This minimum is visible in all seasons. In May to July and in December the mean values are even positive, which means downward transport at the Equator in $\bar{\omega}^{*}$. At higher altitudes, the kinematic upwelling directly at the Equator is also weaker than the surrounding upwelling at around $10^{\circ} \mathrm{N}$ or $10^{\circ} \mathrm{S}$. In the diabatic vertical velocity field, the minimum at $55 \mathrm{hPa}$ is barely visible. There is a hint of lower values at this location in the monthly means of $\bar{\omega}_{\theta}$ from May to July. In contrast to $\bar{\omega}^{*}$, maximum vertical velocities are located at the Equator in several months in the diabatic representation (e.g., October).
There are also other structures of weaker vertical velocity in the kinematic $\bar{\omega}^{*}$ velocity field. Minima in the downwelling regions are also present in the kinematic vertical velocity. In the SH polar region, a minimum in downwelling is visible from June to September throughout the whole altitude range of the stratosphere at $70^{\circ} \mathrm{S}$. From June to August, this feature is also present in the diabatic vertical velocity field, but there the minimum is less distinct and the downward vertical velocity is higher than in $\bar{\omega}^{*}$. In $\mathrm{NH}$ winter, the minimum vertical velocities are visible at high latitudes polewards from $80^{\circ} \mathrm{N}$. This weaker downwelling occurs in the kinematic $\bar{\omega}^{*}$ from November to February. In the zonal mean of $\bar{\omega}_{\theta}$, the minimum at the pole is less pronounced and lasts only from December to January.

In the regions around the addressed minima in the vertical velocity pattern of $\bar{\omega}^{*}$, the surrounding areas often show higher vertical velocities than the diabatic $\bar{\omega}_{\theta}$. One example for the upwelling regions is the monthly mean for February. In $\bar{\omega}^{*}$, there are higher vertical velocities around the equatorial minimum at $55 \mathrm{hPa}$ than in $\bar{\omega}_{\theta}$. At higher altitude at $12 \mathrm{hPa}$ around the second equatorial minimum, there are also high vertical velocities in $\bar{\omega}^{*}$. Here, the effect is most pronounced in the $\mathrm{NH}$, where the kinematic upwelling is about 10 Pa day $^{-1}$ higher than the diabatic upwelling.

Another difference that is clearly visible in the annual mean is the wider upwelling region of the diabatic $\bar{\omega}_{\theta}$ below $700 \mathrm{~K}$. This feature is present in all monthly means throughout the year. 

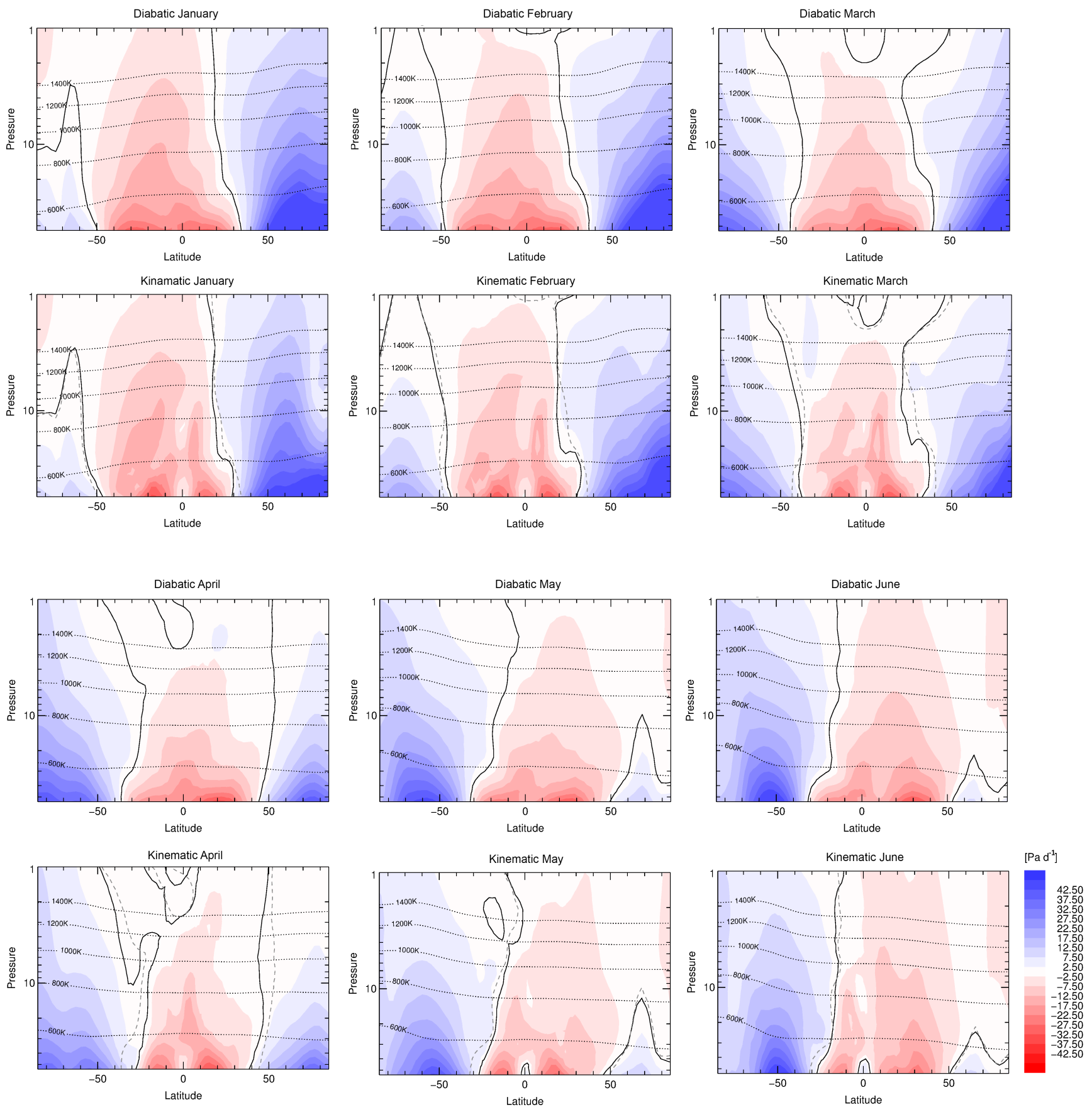

Figure B1. Vertical velocities $\bar{\omega}_{\theta}$ from diabatic heating rates in $\left(\mathrm{Paday}^{-1}\right)$ and transformed Eulerian mean (TEM) vertical velocity $\bar{\omega}^{*}$ in $\left(\right.$ Pa day $\left.^{-1}\right)$ from the 10-year EMAC climatology for the months January-June. 

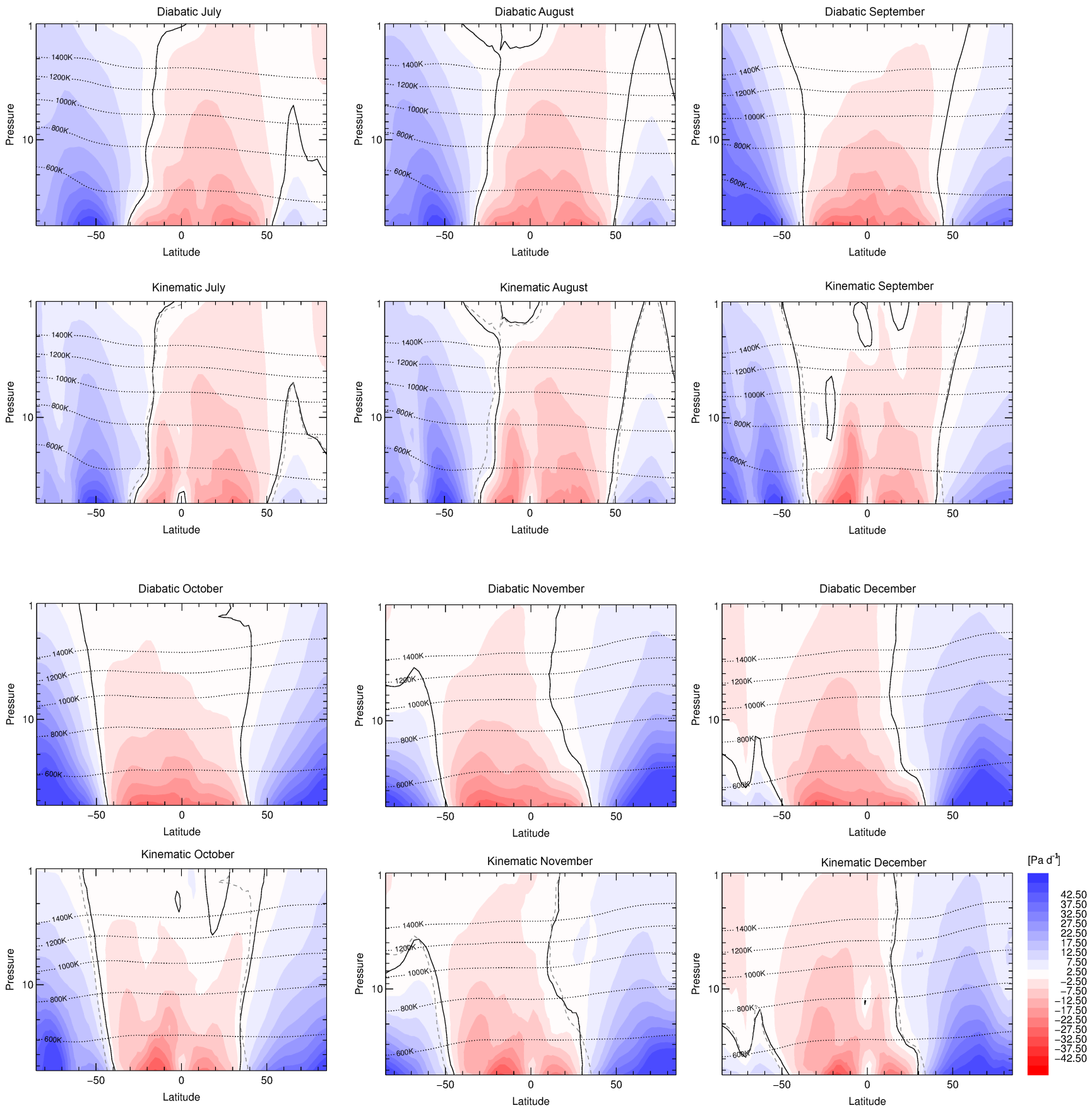

Figure B2. Vertical velocities $\bar{\omega}_{\theta}$ from diabatic heating rates in $\left(\mathrm{Paday}^{-1}\right)$ and transformed Eulerian mean $(\mathrm{TEM})$ vertical velocity $\bar{\omega}^{*}$ in $\left(\right.$ Pa day $\left.^{-1}\right)$ from the 10-year EMAC climatology for the months July-December. 
Acknowledgements. The authors thank P. Jöckel and N. Thomas for their support while working with the models EMAC and CLaMS. We also acknowledge F. Schaps for support in implementing the vertical velocity transformations. We thank S. Rast for providing the tool to obtain the diabatic components from ECHAM6. We acknowledge the Jülich Supercomputing Center (JSC) at Forschungszentrum Jülich for providing computing time and support (project number JIEK71).

The article processing charges for this open-access publication were covered by a Research Centre of the Helmholtz Association.

Edited by: M. Dameris

\section{References}

Abalos, M., Legras, B., and Ploeger, F., and Randel, W. J.: Evaluating the advective Brewer-Dobson circulation in three reanalyses for the period 1979-2012, J. Geophys. Res., 120, 7534-7554, doi:10.1002/2015JD023182, 2015.

Andrews, D. G., Holton, J. R., and Leovy, C. B.: Middle Atmosphere Dynamics, Academic Press, San Diego, 1987.

Bechtold, P., Chaboureau, J., Beljaars, A., Betts, A., Kohler, M., Miller, M., and Redelsperger, J.: The simulation of the diurnal cycle of convective precipitation over land in a global model, $\mathrm{Q}$. J. Roy. Meteor. Soc., 130, 3119-3137, doi:10.1256/qj.03.103, 2004.

Brasseur, G. P., Orlando, J. J., and Tyndall, G. S. (Eds.): Atmospheric Chemistry and Global Change, Oxford University Press, Oxford, 1999.

Butchart, N., Scaife, A. A., Bourqui, M., de Grandpre, J., Hare, S. H. E., Kettleborough, J., Langematz, U., Manzini, E., Sassi, F., Shibata, K., Shindell, D., and Sigmond, M.: Simulations of anthropogenic change in the strength of the Brewer-Dobson circulation, Clim. Dynam., 27, 727-741, doi:10.1007/s00382-006-0162-4, 2006.

Carpenter, R. L., Droegemeier, K. K., Woodward, P. R., and Hane, C. E.: Application of the Piecewise Parabolic Method (PPM) to meteorological modeling, Mon. Weather Rev., 118, 586-612, 1990.

Danielsen, E. F.: Trajectories: isobaric, isentropic and actual, J. Meteorol., 18, 479-486, 1961.

Dee, D. P., Uppala, S. M., Simmons, A. J., Berrisford, P., Poli, P., Kobayashi, S., Andrae, U., Balmaseda, M. A., Balsamo, G., Bauer, P., Bechtold, P., Beljaars, A. C. M., van de Berg, L., Bidlot, J., Bormann, N., Delsol, C., Dragani, R., Fuentes, M., Geer, A. J., Haimberger, L., Healy, S. B., Hersbach, H., Hólm, E. V., Isaksen, L., Kållberg, P., Köhler, M., Matricardi, M., McNally, A. P., Monge-Sanz, B. M., Morcrette, J. J., Park, B. K., Peubey, C., de Rosnay, P., Tavolato, C., Thépaut, J. N., and Vitart, F.: The ERA-Interim reanalysis: configuration and performance of the data assimilation system, Q. J. Roy. Meteor. Soc., 137, 553-597, 2011.

Fueglistaler, S., Legras, B., Beljaars, A., Morcrette, J.-J., Simmons, A., Tompkins, A. M., and Uppala, S.: The diabatic heat budget of the upper troposphere and lower/mid stratosphere in ECMWF reanalyses, Q. J. Roy. Meteor. Soc., 135, 21-37, doi:10.1002/qj.361, 2009.

Garny, H., Birner, T., Bönisch, H., and Bunzel, F.: The effects of mixing on age of air, J. Geophys. Res., 119, 7015-7034, doi:10.1002/2013JD021417, 2014.

Grooß, J.-U., Günther, G., Müller, R., Konopka, P., Bausch, S., Schlager, H., Voigt, C., Volk, C. M., and Toon, G. C.: Simulation of denitrification and ozone loss for the Arctic winter 2002/2003, Atmos. Chem. Phys., 5, 1437-1448, doi:10.5194/acp-5-14372005, 2005.

Hack, J. J.: Parameterization of moist convection in the National Center for Atmospheric Research community climate model (CCM2), J. Geophys. Res., 99, 5551-5568, doi:10.1029/93JD03478, 1994.

Hall, T. M. and Plumb, R. A.: Age as a diagnostic of stratospheric transport, J. Geophys. Res., 99, 1059-1070, 1994.

Hines, C. O.: Doppler-spread parameterization of gravity-wave momentum deposition in the middle atmosphere. Part 1: Basic formulation, J. Atmos. Sol.-Terr. Phy., 59, 371-386, 1997.

Hoppe, C. M.: A Lagrangian transport core for the simulation of stratospheric trace species in a Chemistry Climate Model, $\mathrm{PhD}$ thesis, Bergische Universität Wuppertal, Wuppertal, Germany, available at: http://elpub.bib.uni-wuppertal.de/servlets/ DocumentServlet?id=4210 (last access: 29 October 2015), 2014.

Hoppe, C. M., Hoffmann, L., Konopka, P., Grooß, J.-U., Ploeger, F., Günther, G., Jöckel, P., and Müller, R.: The implementation of the CLaMS Lagrangian transport core into the chemistry climate model EMAC 2.40.1: application on age of air and transport of long-lived trace species, Geosci. Model Dev., 7, 2639-2651, doi:10.5194/gmd-7-2639-2014, 2014.

Jöckel, P., von Kuhlmann, R., Lawrence, M., Steil, B., Brenninkmeijer, C., Crutzen, P., Rasch, P., and Eaton, B.: On a fundamental problem in implementing flux-form advection schemes for tracer transport in 3-dimensional general circulation and chemistry transport models, Q. J. Roy. Meteor. Soc., 127, 10351052, 2001.

Jöckel, P., Tost, H., Pozzer, A., Brühl, C., Buchholz, J., Ganzeveld, L., Hoor, P., Kerkweg, A., Lawrence, M. G., Sander, R., Steil, B., Stiller, G., Tanarhte, M., Taraborrelli, D., van Aardenne, J., and Lelieveld, J.: The atmospheric chemistry general circulation model ECHAM5/MESSy1: consistent simulation of ozone from the surface to the mesosphere, Atmos. Chem. Phys., 6, 5067-5104, doi:10.5194/acp-6-5067-2006, 2006.

Jöckel, P., Kerkweg, A., Pozzer, A., Sander, R., Tost, H., Riede, H., Baumgaertner, A., Gromov, S., and Kern, B.: Development cycle 2 of the Modular Earth Submodel System (MESSy2), Geosci. Model Dev., 3, 717-752, doi:10.5194/gmd-3-717-2010, 2010.

Kasahara, A.: Various vertical coordinate systems used for numerical weather prediction, Mon. Weather Rev., 102, 509-522, 1974.

Konopka, P., Steinhorst, H., Grooss, J., Günther, G., Müller, R., Elkins, J., Jost, H., Richard, E., Schmidt, U., Toon, G., and McKenna, D.: Mixing and ozone loss in the 1999-2000 Arctic vortex: simulations with the three-dimensional Chemical Lagrangian Model of the Stratosphere (CLaMS), J. Geophys. Res., 109, D02315, doi:10.1029/2003JD003792, 2004.

Lauritzen, P., Ullrich, P., and Nair, R.: Atmospheric transport schemes: desirable properties and a semi-lagrangian view on finite-volume discretizations, in: Numerical Techniques 
for Global Atmospheric Models, edited by: Lauritzen, P., Jablonowski, C., Taylor, M., and Nair, R., Vol. 80 of Lecture Notes in Computational Science and Engineering, Springer, Berlin, Heidelberg, 185-250, doi:10.1007/978-3-642-116407_8, 2011 .

Lin, S. and Rood, R.: Multidimensional flux-form semi-Lagrangian transport schemes, Mon. Weather Rev., 124, 2046-2070, 1996.

Lin, S.-J.: A “vertically Lagrangian” finite-volume dynamical core for global models, Mon. Weather Rev., 132, 2293-2307, 2004.

Mahowald, N., Plumb, R., Rasch, P., del Corral, J., Sassi, F., and Heres, W.: Stratospheric transport in a three-dimensional isentropic coordinate model, J. Geophys. Res., 107, ACH3.1ACH3.14, doi:10.1029/2001JD001313, 2002.

McKenna, D. S., Grooß, J.-U., Günther, G., Konopka, P., Müller, R., Carver, G., and Sasano, Y.: A new Chemical Lagrangian Model of the Stratosphere (CLaMS) 2. Formulation of chemistry scheme and initialization, J. Geophys. Res., 107, ACH4.1ACH4.14, doi:10.1029/2000JD000113, 2002a.

McKenna, D. S., Konopka, P., Grooß, J.-U., Günther, G., Müller, R., Spang, R., Offermann, D., and Orsolini, Y.: A new Chemical Lagrangian Model of the Stratosphere (CLaMS) 1. Formulation of advection and mixing, J. Geophys. Res., 107, ACH15.1ACH15.15, doi:10.1029/2000JD000114, 2002b.

Nordeng, T.: Extended Versions of the Convective Parametrization Scheme at ECMWF and Their Impact on the Mean and Transient Activity of the Model in the Tropics, ECMWF technical memorandum, European Centre for Medium-Range Weather Forecasts, available at: http://books.google.de/books? id=ozdmHQAACAAJ (last access: 29 October 2015), 1994.

Paul, J., Fortuin, F., and Kelder, H.: An ozone climatology based on ozonesonde and satellite measurements, J. Geophys. Res., 103, 31709-31734, doi:10.1029/1998JD200008, 1998.

Ploeger, F., Konopka, P., Günther, G., Grooß, J.-U., and Müller, R.: Impact of the vertical velocity scheme on modeling transport across the tropical tropopause layer, Geophys. Res. Lett., 42, 18, doi:10.1002/2014GL062927, 2010.

Ploeger, F., Fueglistaler, S., Grooß, J.-U., Günther, G., Konopka, P., Liu, Y.S., Müller, R., Ravegnani, F., Schiller, C., Ulanovski, A., and Riese, M.: Insight from ozone and water vapour on transport in the tropical tropopause layer (TTL), Atmos. Chem. Phys., 11, 407-419, doi:10.5194/acp-11-407-2011, 2011.

Ploeger, F., Abalos, M., Birner, T., Konopka, P., Legras, B., Müller, R., and Riese, M.: Quantifying the effects of mixing and residual circulation on trends of stratospheric mean age of air, J. Geophys. Res., 42, 2047-2054, doi:10.1002/2014GL062927, 2015.

Pommrich, R., Müller, R., Grooß, J.-U., Konopka, P., Ploeger, F., Vogel, B., Tao, M., Hoppe, C. M., Günther, G., Spelten, N., Hoffmann, L., Pumphrey, H.-C., Viciani, S., D’Amato, F., Volk, C. M., Hoor, P., Schlager, H., and Riese, M.: Tropical troposphere to stratosphere transport of carbon monoxide and long-lived trace species in the Chemical Lagrangian Model of the Stratosphere (CLaMS), Geosci. Model Dev., 7, 2895-2916, doi:10.5194/gmd-7-2895-2014, 2014.
Randel, W. J., Garcia, R. R., and Wu, F.: Dynamical balances and tropical stratospheric upwelling, J. Atmos. Sci., 65, 3584-3595, doi:10.1175/2008JAS2756.1, 2008.

Roeckner, E., Bäuml, G., Bonaventura, L., Brokopf, R., Esch, M., Giorgetta, M., Hagemann, S., Kirchner, I., Kornblueh, L., Manzini, E., Rhodin, A., Schlese, U., Schulzweida, U., and Tompkins, A.: The atmospheric general circulation model ECHAM5. PART I: Model description, Tech. Rep. MPIReport 349, Max Planck Institute for Meteorology, available at: http://www.mpimet.mpg.de/fileadmin/publikationen/Reports/ max_scirep_349.pdf (last access: 29 October 2015), 2003.

Röckner, E., Brokopf, R., Esch, M., Giorgetta, M., Hagemann, S., Kornblueh, L., Manzini, E., Schlese, U., and Schulzweida, U.: Sensitivity of simulated climate to horizontal and vertical resolution in the ECHAM5 atmosphere model, J. Climate, 19, 37713791, 2006.

Schoeberl, M. R., Douglass, A. R., Zhu, Z. X., and Pawson, S.: A comparison of the lower stratospheric age spectra derived from a general circulation model and two data assimilation systems, J. Geophys. Res., 108, 4113, doi:10.1029/2002JD002652, 2003.

Seviour, W. J. M., Butchart, N., and Hardiman, S. C.: The BrewerDobson circulation inferred from ERA-Interim, Q. J. Roy. Meteor. Soc., 138, 878-888, doi:10.1002/qj.966, 2012.

Stevens, B., Giorgetta, M., Esch, M., Mauritsen, T., Crueger, T., Rast, S., Salzmann, M., Schmidt, H., Bader, J., Block, K., Brokopf, R., Fast, I., Kinne, S., Kornblueh, L., Lohmann, U., Pincus, R., Reichler, T., and Roeckner, E.: Atmospheric component of the MPI-M Earth System Model: ECHAM6, Journal of Advances in Modeling Earth Systems, 5, 146-172, doi:10.1002/jame.20015, 2013.

Tiedtke, M.: A comprehensive mass flux scheme for cumulaus parameterization in large-scale models, Mon. Weather Rev., 117, 1779-1800, 1989.

Tost, H., Jöckel, P., and Lelieveld, J.: Influence of different convection parameterisations in a GCM, Atmos. Chem. Phys., 6, 54755493, doi:10.5194/acp-6-5475-2006, 2006.

Wohltmann, I. and Rex, M.: Improvement of vertical and residual velocities in pressure or hybrid sigma-pressure coordinates in analysis data in the stratosphere, Atmos. Chem. Phys., 8, 265 272, doi:10.5194/acp-8-265-2008, 2008.

Wright, J. S. and Fueglistaler, S.: Large differences in reanalyses of diabatic heating in the tropical upper troposphere and lower stratosphere, Atmos. Chem. Phys., 13, 9565-9576, doi:10.5194/acp-13-9565-2013, 2013.

Zhang, G. and McFarlane, N.: Sensitivity of climate simulations to the parameterization of cumulus convection in the Canadian climate center general-circulation model, Atmos. Ocean, 33, 407446, 1995. 